\title{
Integração de Processos Estocásticos e Teoria de Cópulas em Análise de Risco de Projetos de E\&P
}

João Bosco Marques*

\begin{abstract}
Resumo: Nas últimas duas décadas, a teoria de cópula e os processos estocásticos se têm consagrando em finanças, especialmente as cópulas Arquimedianas e os modelos estocásticos de heterocedasticidade condicional. Nesse contexto, este trabalho apresenta uma metodologia consistente de integração dessas vertentes teóricas, fazendo uso de um simulador construído especialmente para esse propósito, possibilitando que sejam gerados modelos com variáveis relevantes de interesse da análise de riscos de projetos de E\&P (Exploração e Produção). O simulador conta com uma proxy de seis equações para as curvas de produção de um campo de petróleo: (i) função linear crescente para início até o platô de produção de óleo; (ii) função linear constante para o platô de produção de óleo; (iii) função exponencial para o declínio de produção do óleo; (iv) função linear crescente para início até o platô de injeção de água; (v) função sigmoide para a produção de água a partir do breakthrough até o abandono do campo e; (vi) função sigmoide para injeção de água a partir do platô até abandono do campo. O exemplo explorado nesse trabalho abrange dois processos estocásticos (GARCH e ARMA), respectivamente para o preço do petróleo e para o custo de capital médio ponderado, e uma cópula bivariada (Gumbel) para o CAPEX (dispêndio de capital) e OPEX. (custo operacional). Esses modelos são utilizados no cálculo do VPL de um projeto de desenvolvimento considerando os dois regimes fiscais vigentes no Brasil: concessão e partilha de produção. $\mathrm{O}$ objetivo é avaliar a distribuição do VPL (Valor Presente Líquido) de projetos de grande complexidade como os da camada do pré-sal brasileiro em condições de incertezas. Elas se referem ao preço da commodity, à taxa de atratividade, ao CAPEX e OPEX e à curva de óleo. Os resultados são indicados em gráficos especiais e a métrica adotada é o valor em risco ponderado, AVaR.
\end{abstract}

Palavras-Chave: Análise de Risco de Projetos de E\&P. Cópulas. Processos Estocásticos. Valor em Risco Ponderado (AVaR).

\section{Integration of Stochastic Processes and Copula Theory in Analysis Risk on E\&P Project}

Abstract: In the last two decades, the theories of copula and stochastic processes have been explored in finance, especially Archimedean copulas and the conditional heteroscedasticity models. In this context, this work presents a consistent methodology for integrating these theories by using a simulator developed especially for this purpose, allowing models generation with relevant variables of interest risk analysis projects for E\&P (Exploration and Production). The simulator has a proxy of six equations for the production curves: (i) crescent linear function from the start until the oil production plateau; (ii) constant linear function to the oil production plateau; (iii) exponential function to the oil production decline; (iv) crescent linear function from the start to water injection plateau; (v) sigmoid function for the water production from its breakthrough until the field abandon; (vi) sigmoid function for the water injection from plateau until the field abandon. The example explored in this work includes two stochastic processes (GARCH and ARMA), respectively for the oil price and the weighted average cost of capital, and a bivariate copula (Gumbel ) for CAPEX (Capital Expenditure) and OPEX (Operational

\footnotetext{
*Engenheiro Mecânico (UFC), Engenheiro de Petróleo Pleno (Petrobras), Mestre em Engenharia de Petróleo (UNICAMP) e Doutor em Avaliação Econômica de Projetos de E\&P (UNICAMP). E-mail: jbdmarques@gmail.com.
} 
Expenditure). These models are used to calculate the NPV of a petroleum project, considering both tax regimes in Brazil: concession and production sharing contract. The objective of this study is to evaluate the distribution of NPV (Net Present Value) projects of great complexity as those of the Brazilian pre-salt under uncertainty conditions. These refer to the price of the commodity, the rate of return, CAPEX and OPEX and oil curve. The results show in special graphics and the metric adopted is the average value at risk, AVaR.

Keywords: Risk Analysis in E\&P Project. Copulas. Stochastic Processes. Average Value at Risk (AVaR).

Classificação JEL: L71, C46, C52, D81.

\section{Introdução}

Nas últimas três décadas, matemáticos, estatísticos e físicos têm-se debruçado na proposição de funções recursivas que sirvam para prever o comportamento e a estrutura de dependência de variáveis econômicas reportadas em séries temporais. O uso de processos estocásticos em análise de riscos de projetos de E\&P se intensificou a partir do início da década de 1990, implicando uma nova era para a decisão de investimento em condições de incerteza.

De fato, esta abordagem é uma evolução dos critérios de decisão sistematizados inicialmente por autores como Newendorp (1976), Megill (1977) e outros. Apesar dessas sistematizações tradicionais, desde o início da década de 1970 já se discutiam as deficiências do método do VPL como critério de decisão de investimento, uma vez que este subestima em geral os custos de oportunidade.

Dos modelos propostos que buscam valorar os custos de oportunidades em projetos de recursos exauríveis, cujas implicações foram amplamente abordadas por Dixit e Pindyck (1994), Copeland e Antikarov (2001) e outros, o ponto de convergência diz respeito ao tratamento estocástico dado as variáveis fundamentais, entre as quais se destacam: o preço das commodities, o volume da reserva, o valor do investimento e o custo de capital médio ponderado.

Modelos de valoração de opções reais de autores como Brennan e Schwartz (1985) e McDonald e Siegel (1986), tornados populares na indústria do petróleo nas décadas de 1990 e 2000, tratam em geral as incertezas com base nos processos de Ito, deixando um caminho livre para que outros pesquisadores proponham processos diferenciados. 


\section{Revisão Bibliográfica}

Entre as commodities, sem dúvida o petróleo é a que mais recebeu a atenção de econometristas e financistas, justificada por sua importância como principal fonte de energia e por suas implicações em diversas atividades produtivas. Desde os trabalhos relacionados ao preço do petróleo e seu impacto nas economias mundiais, de autores como Hamilton (1983), Burbidge e Harrison (1984), Gisser e Goodwin (1986) e Kilian (2009), os processos estocásticos fizeram parte do embasamento teórico que suportaram as conclusões destes e de outros autores. Em todos esses trabalhos, a observação de Morris Adelman (1993, p. 537) mostrou-se inconteste, ao afirmar que o petróleo é tão importante na economia internacional que as previsões de crescimento econômico são invariavelmente qualificadas com a ressalva: "desde que não haja choque do petróleo".

A teoria de cópula, proposta inicialmente por Sklar em 1959, pode ser explorada em conjunto com os processos estocásticos. O teorema de Sklar se baseia em princípios que remontam dos trabalhos de Fréchet (1951), que afirmam que as distribuições marginais podem ser acopladas via função para descrever uma distribuição multivariada. Por algum motivo, durante muito tempo, o termo cópula não era utilizado e os primeiros trabalhos envolvendo modelos de dependência por meio dessas funções surgiram na década de 1980. Não obstante, foi nas últimas duas décadas que o assunto despertou o interesse em diversas áreas de pesquisa e vem crescendo exponencialmente, sobretudo em finanças. Alguns livros tornaram-se referência, como o de Nelsen (1999 e 2006), de McNeil et. al. (2005) e outros.

\section{Fundamentos teóricos}

\subsection{Processos Estocásticos}

A família de processos autorregressivos e de médias móveis foi popularizada por Box e Jenkins em 1970. Esta família de processos é de variância constante, cuja formulação geral é:

$$
y_{t}=c+\overbrace{\sum_{i=1}^{p} \phi_{i} y_{t-i}}^{\operatorname{AR}(p)}+\overbrace{\sum_{j=0}^{q} \theta_{j} \varepsilon_{t-j}}^{\operatorname{MA}(q)}, \quad \operatorname{com} \theta_{0}=1
$$

onde $\varepsilon_{t}$ são ruídos não correlacionados; $\phi_{i}$ e $\theta_{i}$ são os parâmetros do modelo e $p$ e $q$ ditam as defasagens (ordens) do modelo. Trata-se da composição de um processo autorregressivo $\operatorname{AR}(p)$ com outro de média móvel MA $(q)$. Com o retorno das crises econômicas vivenciadas no final da década de 1990 e de 2000, as distribuições de valores extremos e os processos de 
heterocedasticidade condicional ganharam fôlego nas análises de dependência entre variáveis econométricas. Esses últimos, introduzidos por Engle (1982) e generalizados por Bollerslev (1986), tem a seguinte expressão geral:

$$
\operatorname{GARCH}(p, q) \Rightarrow y_{t}=c+\varepsilon_{t} \quad \text { com } \quad \varepsilon_{t}=\sigma_{t} u_{t} \Rightarrow \sigma_{t}^{2}=\omega+\sum_{i=1}^{p} \beta_{i} \sigma_{t-i}^{2}+\sum_{j=1}^{q} \alpha_{j} \varepsilon_{t-j}^{2}, \quad u_{t} \sim \text { i.i.d }(0,1)
$$

onde $\varepsilon_{t}$ são ruídos não correlacionados, sendo desnecessário que a distribuição do erro seja normal, apenas idêntica e independentemente distribuída. As constantes $c, \omega, \beta_{i}$ e $\alpha_{i}$ são os parâmetros a serem estimados. Alternativas são os modelos assimétricos $\operatorname{EGARCH}(\mathrm{p}, \mathrm{q})$ e GJR $(\mathrm{p}, \mathrm{q})$, heterocedasticidade condicional exponencial e modelo de Glosten, Jagannathan e Runkle, respectivamente, conforme definido em Hamilton (1994).

\subsection{Teoria de Cópula}

De modo sucinto, uma cópula é uma função distribuição conjunta de $n$ hipercubos [0,1]. Sklar (1959) demonstrou que qualquer distribuição de probabilidade multivariada $F$ de algum vetor aleatório $X=\left\{X_{1}, \ldots, X_{n}\right\}$ pode ser representada com ajuda de uma função $C$ com a seguinte estrutura:

$$
F\left(x_{1}, \ldots, x_{n}\right)=C\left[P\left(X_{1} \leq x_{1}\right), \ldots, P\left(X_{n} \leq x_{n}\right)\right]=C\left[F_{1}\left(x_{1}\right), \ldots, F_{n}\left(x_{n}\right)\right]=C\left(u_{1}, \ldots, u_{n}\right)
$$

onde $F_{i}\left(x_{i}\right), i=1, \ldots, n$ representam as distribuições marginais. Mais exatamente, uma cópula de dimensão $n$ é uma função de distribuição conjunta de $n$ variáveis aleatórias com distribuição uniforme Unif(0,1), obtida a partir das distribuições marginais subjacentes e satisfaz as seguintes condições para todo $i \leq n$ e $a_{n} \in[0,1]$ : (i) $C\left(1, \ldots, 1, a_{i}, 1, \ldots, 1\right)=a_{i} ;$, (ii) $C\left(a_{1}, \ldots, a_{n}\right)=0$ se $a_{i}=0$ e; (iii) $C$ é crescente nas $n$ dimensões. Para cada categoria de cópula há diversas classes, mas duas delas são mais populares: as elípticas e as Arquimedianas. Das elípticas as mais comuns são a cópula Gaussiana e a $t$ de Student. Das Arquimedianas são as cópulas clássicas de Gumbel, Clayton e Frank. Por exemplo, a cópula multivariada de Clayton (1978) tem a seguinte expressão:

$$
C^{C l}\left(u_{1}, \ldots, u_{n} ; \theta\right)=\left(u_{1}^{-\theta}+\ldots+u_{n}^{-\theta}-1\right)^{-1 / \theta}
$$

onde $\theta>0$ é o parâmetro que expressa o nível de dependência. Quando $\theta \rightarrow 0$ implica independência e quando $\theta \rightarrow \infty$ implica dependência perfeita positiva (não há dependência negativa). A cópula multivariada de Frank (1979) tem a seguinte formulação: 


$$
C^{F r}\left(u_{1}, \ldots, u_{n} ; \theta\right)=-\frac{1}{\theta} \ln \left[1+\frac{\left(e^{-\theta u_{1}}-1\right) \cdot \ldots \cdot\left(e^{-\theta u_{n}}-1\right)}{e^{-\theta}-1}\right]
$$

onde $-\infty<\theta<\infty$ é o parâmetro que expressa o nível de dependência entre as funções marginais. Quando $\theta \rightarrow 0$ implica independência; $\theta \rightarrow \infty$ implica dependência perfeita positiva e quando $\theta \rightarrow-\infty$ implica dependência perfeita negativa. É uma cópula popular pelo fato de permitir avaliações de dependências simétricas nas duas direções, semelhante à cópula $t$ de Student. A cópula de Gumbel foi apresentada em 1960, cuja expressão multivariada é

$$
C^{G u}\left(u_{1}, \ldots, u_{n} ; \theta\right)=e^{\left\{-\left[\left(-\ln u_{1}\right)^{\theta}+\ldots+\left(-\log u_{n}\right)^{\theta}\right]^{1 / \theta}\right\}}
$$

onde $\theta \geq 1$ é o parâmetro que expressa o nível de dependência. Quando $\theta=1$ indica independência e $\theta \rightarrow \infty$ indica dependência perfeita positiva e não ha dependência perfeita negativa. Esta cópula contrasta com a de Clayton, pois exibe fraca dependência entre as caudas inferiores e é fortemente correlacionada entre as caudas superiores. Na Figura 1 a seguir estão ilustradas as cópulas Arquimedianas clássicas.
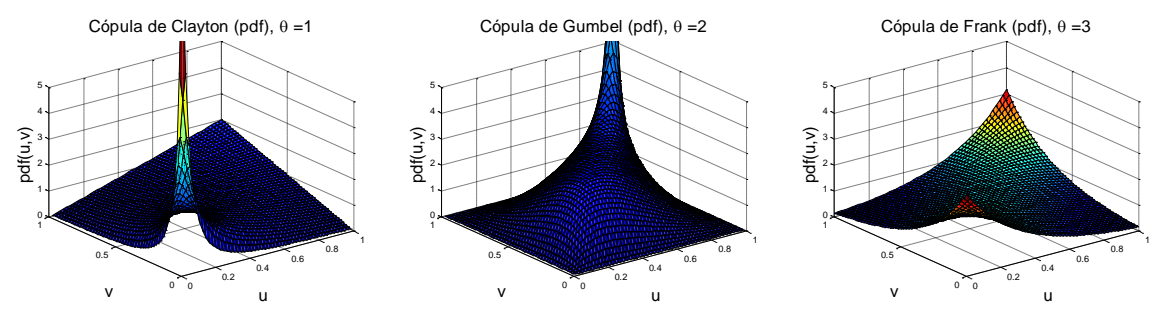

Figura 1: Cópulas Arquimedianas clássica: Clayton, Frank e Gumbel

Uma das principais motivações para analisar uma cópula é que ela permite avaliar a dependência geral entre as variáveis aleatórias, pois ao transformar as variáveis $X_{1}$ e $X_{2}$ para $U$ e $V$, por exemplo, uma cópula bivariada herda informação da distribuição conjunta que não está contida nas distribuições marginais. Por este motivo, Nelsen (2006) revela que uma cópula é chamada algumas vezes de função de dependência, cujas vantagens são: (i) a modelagem da dependência é mais geral; (ii) a cópula é invariante para as transformações monotônicas crescentes e; (iii) a estimação e a inferência são baseadas em métodos de máxima verossimilhança, cujos procedimentos são fáceis e disponíveis em softwares comerciais. 


\subsection{Risco}

Por definição, o VaR expressa um nível mínimo de perda em valor financeiro (ou em temos de retorno) com alto nível de confiança (em geral de 95\% a 99\%) para um horizonte de tempo pré definido. Por exemplo, se o retorno de um projeto de \$1 bilhão tem um VaR de 99\% de confiança igual a $2 \%$ para um horizonte de um dia, implica dizer que a probabilidade do investidor sofrer um prejuízo mínimo de \$20 MM em um dado dia é de 1\%. Em notação estatística, para um variável aleatória $X$ qualquer, o VaR em um nível de confiança (1- $\alpha) 100 \%$ é definido como:

$$
\operatorname{VaR}_{\alpha}(X)=-\inf \{x \mid P(X \leq x) \geq \alpha\}=-F_{X}^{-1}(\alpha)
$$

onde $\alpha \in(0,1)$ e $F_{X}{ }^{-1}(\alpha)$ é o inverso da função distribuição da variável $X$. A desvantagem desta métrica é que eventos extremos não são refletidos. Por exemplo, nada se pode afirmar em relação aos percentuais menores que $1 \%$ ou $5 \%$, que dependendo da distribuição pode representar perdas ou ganhos extraordinários. O mais indicado seria utilizar uma métrica que reflita todo o conteúdo de interesse da distribuição, considerando os valores de perdas para níveis de confiança superiores a 95\%, por exemplo. Essa é a proposta da métrica AVaR, o valor em risco ponderado, também conhecido pelo termo valor em risco condicional (CVaR). Conforme Rockafellar e Uryasev (2000), o AVaR é definido como:

$$
\operatorname{AVaR}_{\alpha}(X)=\frac{1}{\alpha} \int_{0}^{\alpha} \operatorname{VaR}_{p}(X) d p
$$

onde VaR é determinado pela Eq. (7). De acordo com a Eq. (8), o AVaR com probabilidade $\alpha$ é definido como a média dos $V a R$ 's de probabilidade $\alpha$ com graus de confiança maiores que (1$\alpha) \%$. A medida $A V a R$ evita, a depender da distribuição, subestimação ou superestimação do risco, como ocorre em geral com as medidas de risco com base nos percentis P10, P50 e P90, comumente utilizada em estudos de reservatórios. Uma interpretação geométrica do $A V a R$ pode ser dada pela inversa da função distribuição $F_{X}{ }^{-1}$ e pela função densidade $f(x)$, ver Figura 2 a seguir: 


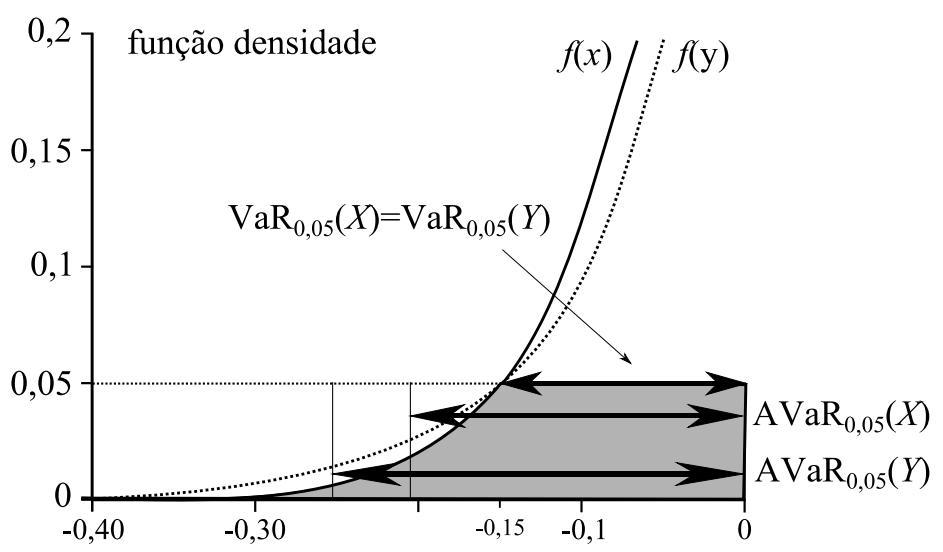

Figura 2: Função inversa $F_{X}{ }^{-1}$ e a função densidade, $f(x)$ - conceito de $A V a R$.

$\mathrm{O} A \operatorname{VaR}_{0,05}(X)$ é um número tal que, tomando-se um retângulo com altura 0,05 o produto $0,05 \times A \operatorname{VaR}_{0,05}(X)$ resulta o valor da área sombreada no gráfico da função densidade. Note-se que $A \operatorname{VaR}_{0,05}(X)>A \operatorname{VaR}_{0,05}(Y)$, embora $\operatorname{VaR}_{0,05}(X)=\operatorname{VaR}_{0,05}(Y)$. Nesse caso específico, a medida VaR subestima o risco. Razão pela qual indicamos a métrica AVaR.

\section{Metodologia de trabalho}

A premissa fundamental para esta metodologia é a existência de uma estratégia de produção, de preferência oriunda de simulação de reservatórios de petróleo. Para este trabalho foi desenvolvido um programa em MatLab ${ }^{\circledR}$ para viabilizar a integração de processos estocásticos e a teoria de cópulas na avaliação do risco de um projeto decorrente de uma estratégia de produção selecionada como premissa. Esta metodologia consiste de 7 etapas conforme o esquema indicado na Figura 1.

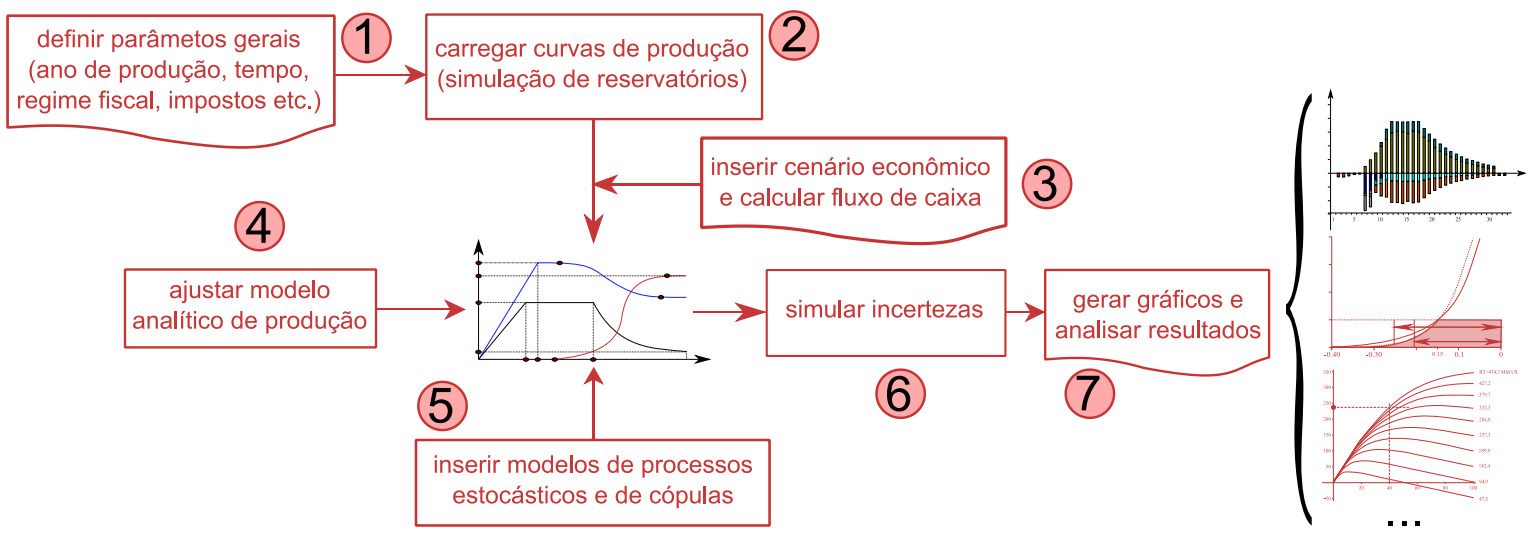

Figura 1: Esquema simplificado das etapas da metodologia de integração. 


\section{Etapa 1}

A primeira etapa consiste em definir os parâmetros gerais para o cálculo do fluxo de caixa de um projeto de E\&P. Esses parâmetros são: regime fiscal (concessão e partilha), início da produção (2010), tempo de produção (30 anos), impostos e contribuições governamentais, parâmetros de partilha de produção (óleo custo, óleo lucro, custos a recuperar, percentual de partilha etc.).

\section{Etapa 2}

Esta etapa consiste em carregar as curvas da estratégia de produção selecionada: como premissa. As curvas são: óleo, gás, injeção e produção de água.

\section{Etapa 3}

A inserção dos parâmetros econômicos é realizada através de um módulo do programa que permite criar/salvar quaisquer cenários econômicos. Para este ensaio, especificamente, foram considerados os valores descritos na Tabela 1.

Tabela 1: Cenários econômicos

\begin{tabular}{|c|c|c|c|c|}
\hline Variável & Otimista & Referência & Pessimista & Unidade \\
\hline Preço do óleo & 104 & 80 & 56 & U\$/bbl \\
\hline Preço do gás & 9,1 & 7,0 & 4,9 & U\$/MM Btu \\
\hline TMA (taxa mínima de atratividade) & 8,4 & 12,0 & 15,6 & $\%$ \\
\hline CAPEX & & & & - \\
\hline $\begin{array}{r}\text { Despesas iniciais (estudos de } \\
\text { reservatórios) }\end{array}$ & 84 & 120 & 156 & U\$ MM \\
\hline Modelo de custo de plataforma & Mod. 1 & Mod. 2 & Mod. 3 & U\$ MM \\
\hline Custo médio por poço & 42 & 60 & 78 & U\$ MM/poço \\
\hline OPEX & & & & - \\
\hline Custo fixo & 24,5 & 35,0 & 45,5 & U\$ MM/ano \\
\hline Custo variável & & & & - \\
\hline Injeção de fluidos (água) & 4,2 & 6,0 & 7,8 & $\mathrm{U} \$ / \mathrm{bbl}$ \\
\hline Separação de fluidos (óleo, gás e água) & 2,8 & 4,0 & 5,2 & $\mathrm{U} \$ / \mathrm{bbl}$ \\
\hline Descarte de fluidos (água produzida) & 2,1 & 3,0 & 3,9 & U\$/bbl \\
\hline
\end{tabular}

O cálculo do custo da plataforma (MM U\$) é ditado de acordo com a seguinte métrica:

$$
\mathrm{CAPEX}_{\text {plat }}=k_{1} \times \frac{1}{1000} \operatorname{máx}\left[Q_{t, \text { lleo }}^{m^{3} / d i a}+Q I_{t}^{m^{3} / d i a}+Q W_{t}^{m^{3} / d i a}\right]+k_{2} \quad\left\{\begin{array}{lll}
\text { Mod.1 } & k_{1}=5,3 & k_{2}=371 \\
\text { Mod.2 } & k_{1}=7,6 & k_{2}=530 \\
\text { Mod.3 } & k_{1}=9,9 & k_{2}=689
\end{array}\right.
$$


onde $Q_{o}$ é a vazão de óleo e $W_{i+p}$ é a soma das vazões de água injetada e produzida. Os dados econômicos da Tabela 1 e a Eq. (1) são consistentes para projetos offshore com lâmina d'água maior que $1000 \mathrm{~m}$ e distante da costa entre 100 e $300 \mathrm{~km}$. Mais exatamente, são dados econômicos adequados para os projetos do pré-sal brasileiro.

Nesta etapa se calcula também o fluxo de caixa conforme regime fiscal adotado, considerando os aspectos legais que mais impactam o VPL. Este, de modo simplificado, depende fundamentalmente de duas variáveis: o fluxo de caixa líquido apurado ao final de cada ano e a taxa de desconto anual, TMA, ou seja:

$$
V P L_{\text {prod }}=\sum_{i=1}^{n_{p}} \frac{F C L_{i, p r o d}}{(1+T M A)^{i}}
$$

onde $n_{p}$ é o período total de produção. Para o caso de regime de concessão o $F C L$ é

$$
F C L_{t, p r o d}=\overbrace{\left(R_{t}-C O_{t}-P I S_{t}-R O Y_{t}-I_{t, N D}-D_{t}-P E_{t}\right)}^{\text {Lucro tributavd }} \cdot(1-I R-C S L L)+D_{t}-I_{t, D}
$$

onde $R$ é o rendimento bruto; $C O$ é o custo operacional (fixo e variável); PIS são as contribuições sociais (PIS, PASEP e COFINS, 9,25\%); ROY são os royalties (10\%); $I_{N D}$ é o investimento não depreciável; $D$ é a depreciação; $P E$ é a participação especial; $I R$ é o imposto de renda (25\%); CSLL é a contribuição social sobre o lucro líquido (9\%); $I_{D}$ é o investimento depreciável e o subscrito $t$ refere-se ao ano fiscal. O montante $I_{D}$ incide no ano 0 e o $I_{N D}$ nos anos 0 e 1 (as despesas iniciais dos estudos de reservatórios incidem no ano 0 ). $\mathrm{O}$ rendimento bruto $R$ é o resultado da produção de óleo e gás multiplicada por suas respectivas cotações, ou seja:

$$
R_{t}=Q_{t, \text { óleo }} \times p_{t, \text { óleo }}+Q_{t, g a ́ s} \times p_{t, \text { gás }}
$$

onde $Q_{\text {óleo }}$ é expresso em $b b l /$ ano, $Q_{\text {gás }}$ em MM BTU/ano, $p_{\text {óleo }}$ em U $\$ / b b l$ e $p_{\text {gás }}$ em U\$/MM BTU.

A depreciação é linear e incide sobre o investimento depreciável $I_{D}$ (plataforma). Para o caso de regime fiscal de contrato de partilha de produção, a função que define o fluxo de caixa é

$$
F C L_{t, \text { prod }}=[(1-G) \overbrace{\left(R_{t}-R O Y_{t}-C_{t, \text { rec }}\right)}^{\text {Óloolucro(profit oil) }}] \cdot(1-\mathrm{IR}-\mathrm{CSLL})+D_{t}-I_{i, D}
$$

onde $G$ é o percentual devido ao governo por contrato de partilha $(41,65 \%), R O Y$ são os royalties (15\%) e $C_{r e c}$ é o custo a recuperar. Por exemplo, para o contrato de exploração do campo de Libra, o percentual $G$ é de $41,65 \%$ e o custo a recuperar é de no máximo $50 \%$ do 
faturamento bruto para os anos 1 e 2 e, para os anos seguintes, o custo a recuperar é de no máximo 30\%. Logo

$$
\left(\mathrm{OPEX}_{t, \text { total }}+I_{t, N D}\right) \leq C_{t, \text { rec }} \leq 0,5 R_{t}(\text { ano } 1 \mathrm{e} 2) \text { e } 0,3 R_{t}(\text { ano } 3 \mathrm{em} \text { diante })
$$

\section{Etapa 4}

As curvas analíticas de produção e injeção de fluidos segue o esquema indicado na Figura 2 a seguir:
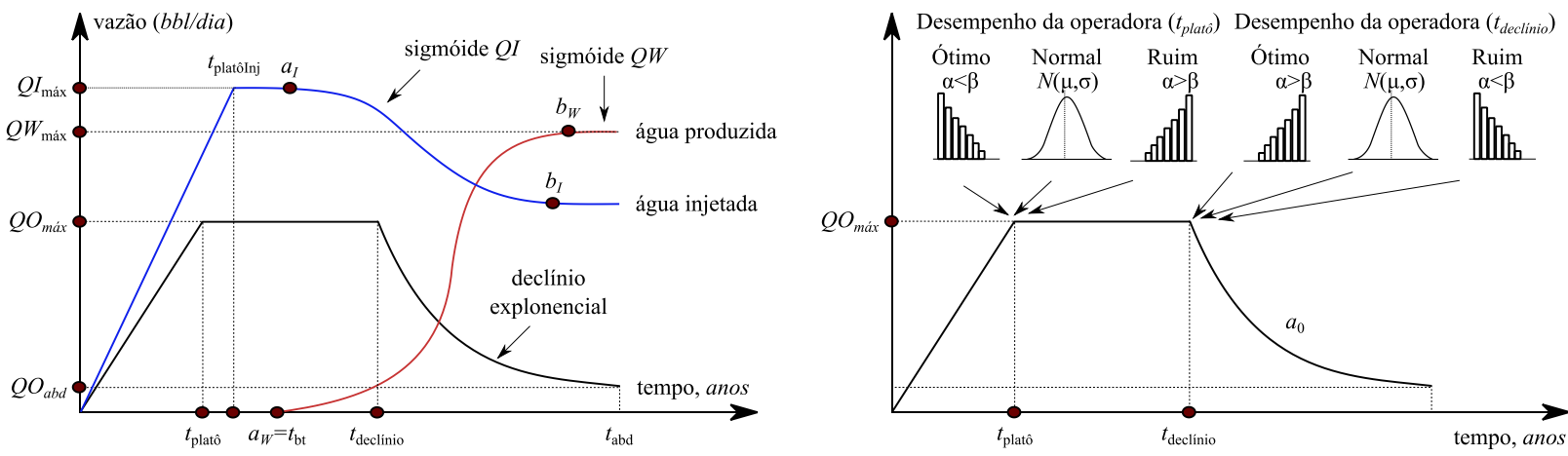

Figura 2: Curvas de produção do reservatório e desempenho da operadora.

As curvas do gráfico (A) são: (i) função linear crescente para início da produção de óleo até o platô; (ii) função linear constante para manutenção do platô de produção de óleo; (iii) função exponencial para o declínio de produção de óleo; (iv) função linear crescente para o início de injeção de água até seu valor máximo; $(v)$ função sigmoide para a produção de água a partir do breakthrough até abandono do campo e; (vi) função sigmoide para a injeção de água a partir da vazão máxima de injeção até o abandono do campo. Em termos de equações tem-se o seguinte esquema:

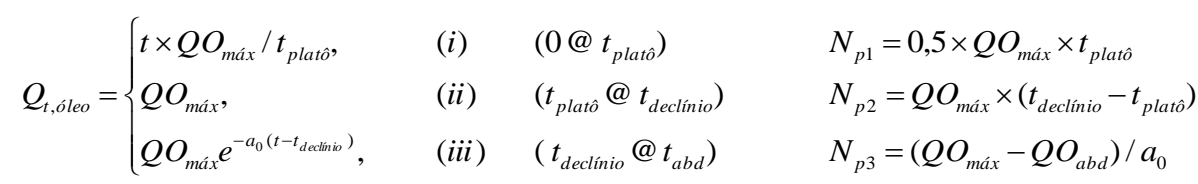

onde $a_{0}$ é a taxa de declínio $\left(t^{-1}\right)$ e a reserva de óleo é data por $N_{p}=N_{p 1}+N_{p 2}+N_{p 3}$. Para a curva de produção de água do campo tem-se que

$$
\text { (v) } Q W_{t}\left(0 @ t_{a b d}\right)=Q W_{\max } \times \begin{cases}0, & 0 \leq t \leq a_{W}=t_{b t} \\ 2\left[\left(t-t_{b t}\right) /\left(b_{W}-t_{b t}\right)\right]^{2}, & t_{b t} \leq t \leq\left(t_{b t}+b_{W}\right) / 2 \\ 1-2\left[\left(t-t_{b t}\right) /\left(b_{W}-t_{b t}\right)\right]^{2}, & \left(t_{b t}+b_{W}\right) / 2 \leq t \leq b_{W} \\ 1, & b_{W} \leq t \leq t_{a b d}\end{cases}
$$


onde $a_{W}$ e $b_{W}$ são parâmetros da função sigmoide da curva de produção de água. O sistema de equações para a injeção de água é:

$$
\begin{aligned}
& \text { (iv) } Q I_{t}\left(0 @ t_{\text {platôthj }}\right)=t \times Q I_{\text {max }} / t_{\text {platôthj; }}
\end{aligned}
$$

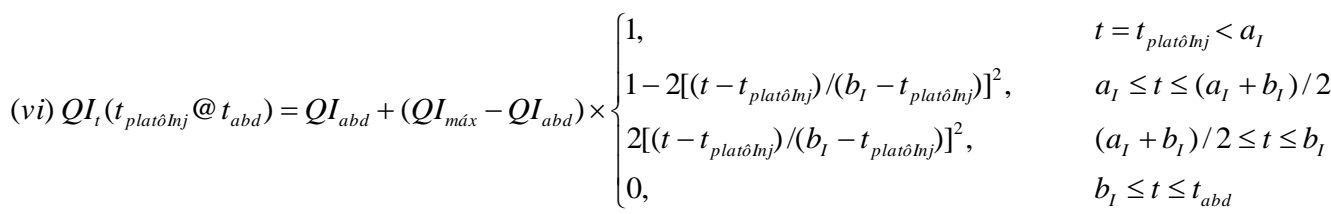

onde $a_{I}$ e $b_{I}$ são os parâmetros da função sigmoide da curva de injeção de água. A vazão de gás é função da razão gás/óleo, ou seja, $Q_{g a ́ s}=Q_{\text {óleo }} \times \mathrm{RGO}$ (aqui foi utilizado um $\mathrm{RGO}=250 \mathrm{~m}^{3} / \mathrm{m}^{3}$ std, razão gás/óleo), implicando uma produção acumulada de gás $G_{p}=\mathrm{RGO} \times N_{p}$. O programa dispõe de um algoritmo que permite ajustar as curvas do modelo analítico contra as curvas da estratégia de produção. Este ajuste é feito alterando os valores das variáveis contidas no seguinte conjunto:

$$
\{\overbrace{\left\{t_{\text {platô }}, t_{\text {declínio }}, Q O_{\text {maxx }}, a_{0}\right.}^{C_{\text {model }}=\text { varíaveismais sensíveis }}, t_{b t}, t_{\text {platồnj }}, Q O_{a b d}, Q W_{\text {máx }}, Q I_{\text {máx }}, Q I_{a b d}, b_{W}, a_{I}, b_{I})
$$

O ajuste é realizado até que se obtenha um erro máximo de $2 \%$ entre as curvas do modelo analítico e da estratégia de produção selecionada. Note-se também que, de acordo com a Figura 2 (B), uma operadora tem desempenho ótimo se executar o projeto antecipando o tempo de platô de produção e/ou postergando o tempo de declínio em relação aos tempos previstos na estratégia de produção. Deste modo, é possível gerar $n$ conjuntos de curvas por simulação e avaliar a distribuição do $N_{p}$ (reserva) considerando as incertezas das variáveis mais sensíveis em (10), conforme o desempenho da operadora na execução do projeto (ótimo, normal, ruim).

\section{Etapas 5 e 6}

A Etapa 5 consiste da elaboração dos processos estocásticos e de cópulas bivariadas. $\mathrm{O}$ módulo de processos estocásticos permite desenvolver modelos $\operatorname{ARMA}(p, q), \operatorname{GARCH}(p, q)$, $\operatorname{EGARCH}(p, q), \operatorname{GJR}(p, q)$ e alguns processos de Ito: MAB, MGB, modelo MGB com reversão à média, modelo de Cox-Ingersoll-Ross etc. $\mathrm{O}$ módulo de cópulas permite parametrizar as cópulas Gaussianas, $t$ de Student, Gumbel, Frank e Clayton. A Etapa 6 é a simulação propriamente dita, considerando tanto as incertezas econômicas como as incertezas referentes às curvas de produção geradas conforme o desempenho da operadora. Os modelos de processos estocásticos e de cópulas empregados nesse trabalho estão indicados na Tabela 2. 
Tabela 2 - Modelos de processos estocásticos e de cópulas - Simulação do fluxo de caixa

\begin{tabular}{ll|llll}
\hline Variável econômica & Modelo proposto & $\begin{array}{l}\text { Curva de } \\
\text { produção }\end{array}$ & Ótimo & Normal & Ruim \\
\hline Preço do óleo & $\operatorname{GARCH}(p, q)$ & $t_{\text {platô }}$ & $\operatorname{Beta}(\alpha, \beta)$ & $N(\mu, \sigma)$ & $\operatorname{Beta}(\alpha, \beta)$ \\
TMA & $\operatorname{ARMA}(p, q)$ & $t_{\text {declinio }}$ & $\operatorname{Beta}(\alpha, \beta)$ & $N(\mu, \sigma)$ & $\operatorname{Beta}(\alpha, \beta)$ \\
OPEX e CAPEX & Cópula bivariada & $Q O_{\text {máx }}$ & Uniforme & Uniforme & Uniforme \\
& & $a_{0}$ & Uniforme & Uniforme & Uniforme \\
\hline
\end{tabular}

\section{Etapa 7}

A última etapa consiste em analisar os resultados gerados a partir das simulações do fluxo de caixa do projeto. Nesse trabalho os resultados são reportados para os dois regimes fiscais, concessão e partilha. Os gráficos são: (i) VPL versus CAPEX e OPEX, (ii) ROI versus TMA, (iii) Curvas de risco e AVaR (VPL e reserva).

\section{Resultados}

A escolha do processo GARCH para representar o preço da commodity petróleo é justificada, uma vez que as séries históricas de retornos da cotação apresentam períodos de diferentes variâncias (ver Figura 3).
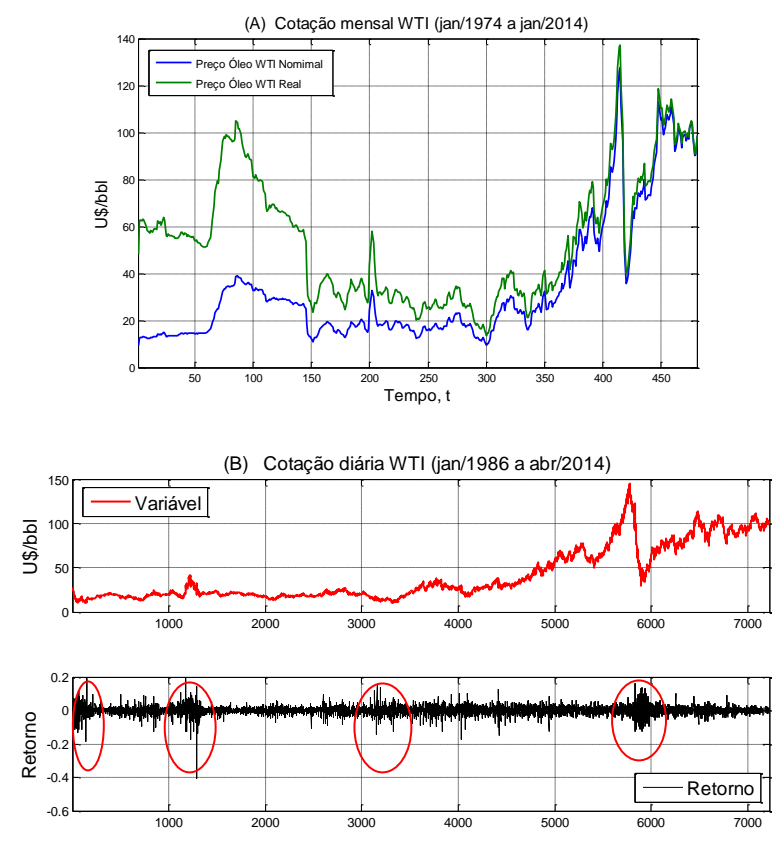

Figura 3: (A) cotação WTI mensal real e nominal, (B) cotação WTI diária nominal. 
O efeito de heterocedasticidade é verificado tanto para a cotação real como a nominal. Para a análise de risco indicada nesse trabalho foi ajustado um modelo $\operatorname{GARCH}(2,1)$ com base na cotação mensal WTI (West Texas Intermediate, valor real) entre out/2000 a jan/2014 (160 amostras).
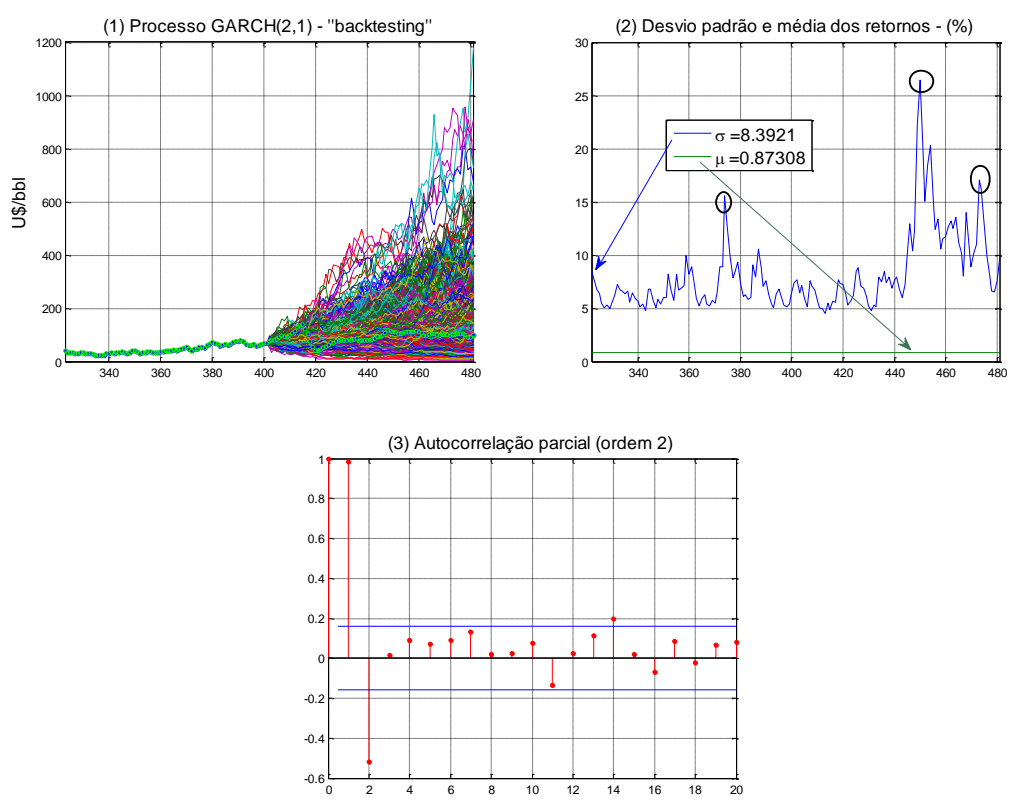

Figura 4: Análise - simulação $\operatorname{GARCH}(2,1)$ - cotação WTI, amostras mensais out/2000 a jan/2014.
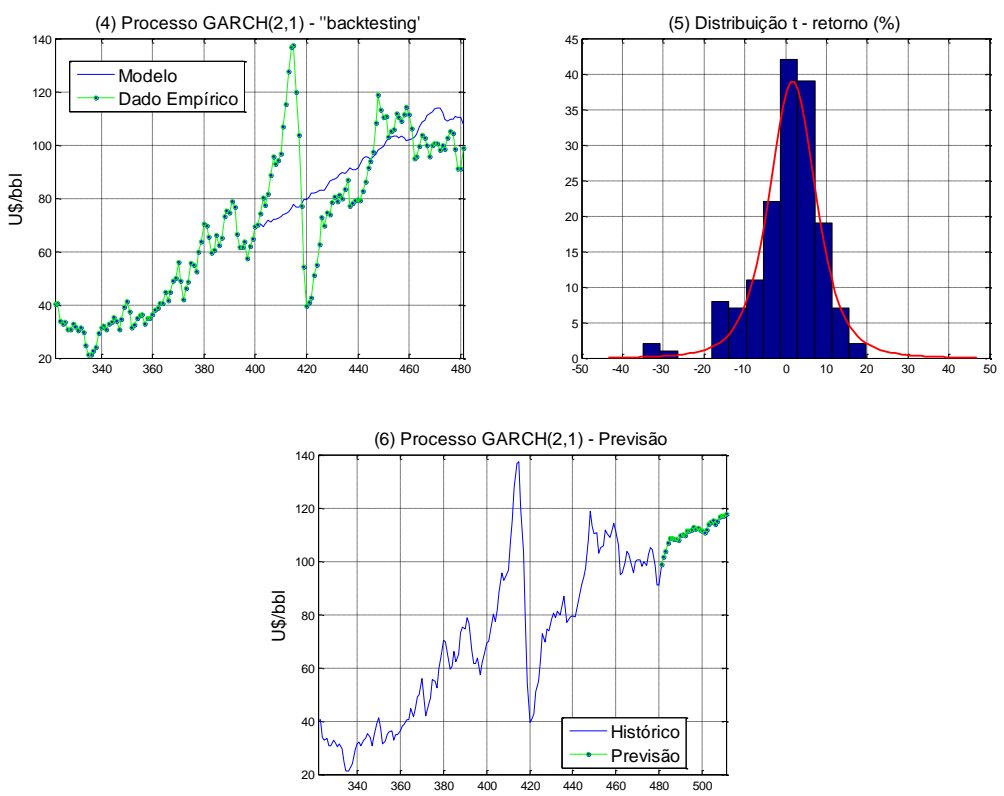

Figura 5: Backtesting e previsão - simulação $\operatorname{GARCH}(2,1)$ - cotação WTI, amostras mensais out/2000 a jan/2014. 
Note-se que a função de autocorrelação parcial de ordem 2 (gráfico 3) apresenta um valor persistente até a segunda defasagem, indicando um possível modelo GARCH com até dois coeficientes autorregressivo ( $\beta_{1}$ e $\beta_{2}$ ) para a variância. O programa desenvolvido permite a realização de um backtesting (gráficos 1 e 4) onde os coeficientes do modelo são inferidos com base na primeira metade das amostras e os valores previstos são comparados com a segunda metade. Na Tabela 3 estão indicados os coeficientes dos modelos GARGH e ARMA:

Tabela 3: Modelo GARCH(2,1) para a commodity petróleo e ARMA $(3,1)$ para a TMA

\begin{tabular}{|c|c|c|c|c|c|}
\hline \multicolumn{3}{|c|}{ GARCH $(2,1)$ - Preço do petróleo } & \multicolumn{3}{|c|}{ ARMA $(3,1)$ - Taxa Mínima de Atatividade } \\
\hline Coeficientes & Valores & Erro (\%) & Coeficientes & Valores & Erro $(\%)$ \\
\hline$\beta_{1}, \beta_{2}$ & {$\left[\begin{array}{ll}0 & 0,4100\end{array}\right]$} & $\begin{array}{l}{[0,1382} \\
0,1504]\end{array}$ & $\phi_{1}, \phi_{2}$ e $\phi_{3}$ & $\begin{array}{ll}{[0,0107} & 0,4184 \\
0,1474] & \end{array}$ & $\begin{array}{l}{[1,714} \\
0,552]\end{array}$ \\
\hline $\begin{array}{c}\alpha_{1} \\
c \mathrm{e} \omega\end{array}$ & $\begin{array}{l}{[0,5256]} \\
0,5189 \\
5,1386\end{array}$ & {$[0,1568]$} & $\theta_{1}$ & {$[0,2760]$} & {$[1,753]$} \\
\hline
\end{tabular}

A cópula ajustada para o vetor (CAPEX, OPEX) é a cópula de Gumbel. O ajuste desta indicou um parâmetro $\theta=1,9628$ e foram gerados 1000 pares aleatoriamente para uso na simulação (ver Figura 6).
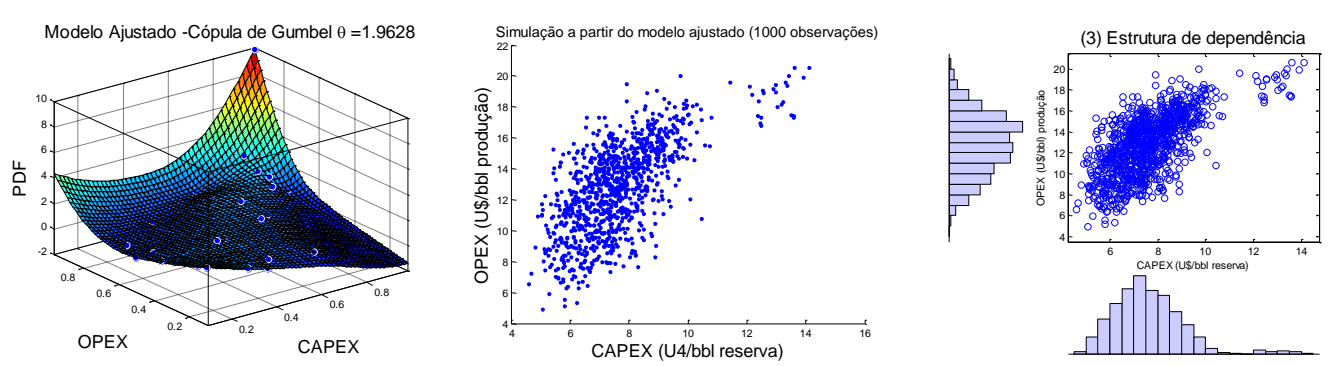

Figura 6: OPEX e CAPEX gerados aleatoriamente a partir da cópula de Gumbel.

Neste trabalho, a análise de risco refere-se ao desenvolvimento de um campo de petróleo com volume recuperável aproximado de $350 \mathrm{MM}$ de $b b l$, cuja estratégia de produção selecionada prevê a perfuração de 18 poços (12 produtores e 6 injetores). O ajuste do modelo analítico proposto nas Eq. (7) a (9) contra as curvas da estratégia de produção está indicado na Figura 7, admitindo-se um erro máximo de $2 \%$ para os volumes acumulados, $N_{p}, G_{p}, W_{p}$ e $W_{i}$. 

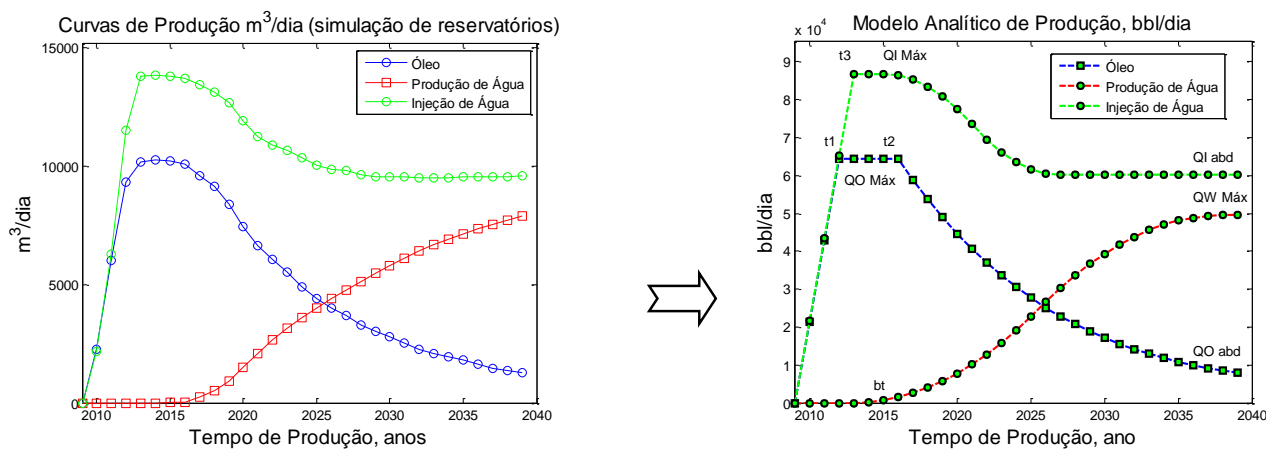

Figura 7: Ajuste das variáveis do sistema de equações contra as curvas da estratégia de produção.

Os resultados deste ajuste e as incertezas assumidas nas simulações para as variáveis mais sensíveis em (10) estão indicados na Tabela 4 a seguir.

Tabela 4: Distribuições das variáveis do vetor $C^{o}{ }_{\text {model, }}$ Eq. (10)

\begin{tabular}{l|ccccccc}
\hline \multicolumn{1}{c|}{ Variável } & Ótimo & Normal & Ruim & mín & $\mu$ & máx & Unidade \\
\hline$t_{\text {platô }}$ & Beta(1,3) & $N(3,2)$ & $\operatorname{Beta}(3,1)$ & 1 & 3 & 5 & ano \\
$t_{\text {declinio }}$ & Beta(3,1) & $N(7,3)$ & Beta $(1,3)$ & 3 & 7 & 10 & ano \\
$Q O_{\text {máx }}$ & Uniforme & Uniforme & Uniforme & 31.414 & 64.828 & 94.242 & $\mathrm{bbl} / \mathrm{dia}$ \\
$a_{0}$ & Uniforme & Uniforme & Uniforme & 0,0293 & 0,0586 & 0,0879 & $t^{-1}$ \\
\hline
\end{tabular}

\section{VPL versus CAPEX e OPEX}

O simulador permite que sejam gerados diversos gráficos. A Figura 8, por exemplo, indica a região de aceitação e rejeição do projeto em função do CAPEX e OPEX para um preço de petróleo de U\$80/bbl e TMA de 12\%. Note-se, nesse caso, que a margem de valores CAPEX e OPEX para aceitação do projeto é maior se o regime é de concessão.
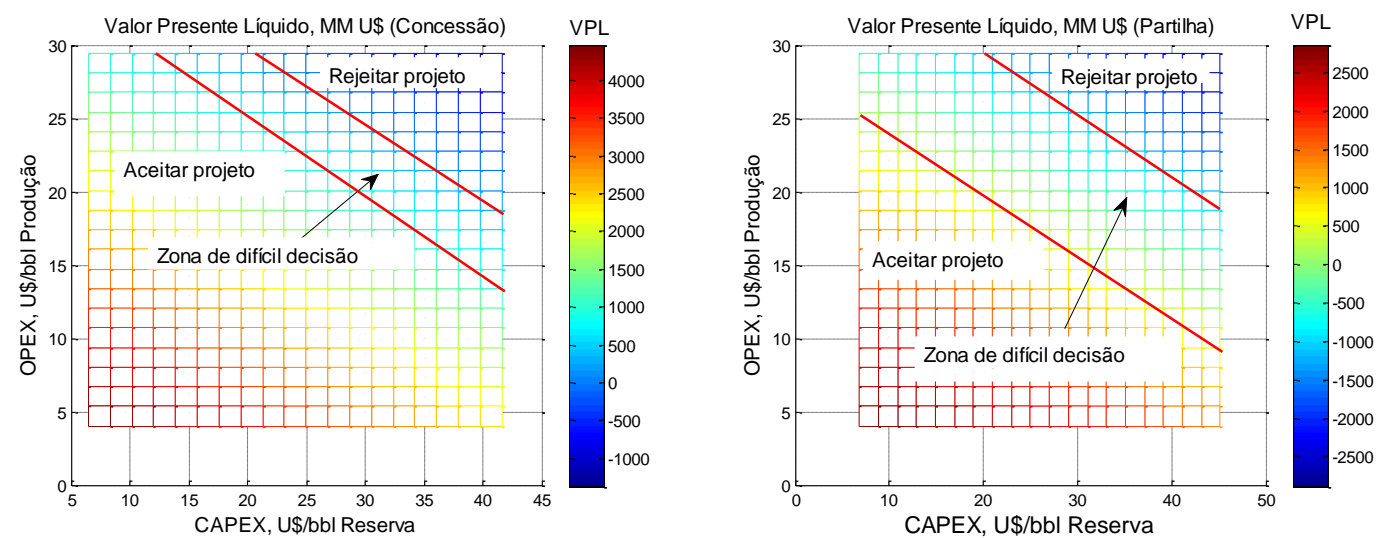

Figura 8: VPL versus OPEX, CAPEX (concessão e partilha de produção). 
É claro que a região de aceitação para o regime de partilha pode, eventualmente, ser maior que a de concessão. Este resultado dependerá basicamente do percentual de custo a recuperar e da partilha definidos no contrato de partilha, conforme Eq. (5) e (6).

\section{ROI versus TMA}

A Figura 9 a seguir indica o ROI (retorno sobre o investimento) em função da taxa de atratividade do projeto para um OPEX constante e vários valores de CAPEX. Por exemplo, para um CAPEX de U\$21,71/bbl em regime de concessão, um retorno mínimo de $20 \%$ sobre o investimento é possível com taxas inferiores a 20\%. Note-se que, para o regime de partilha, este retorno é possível com taxas inferiores a $14 \%$.
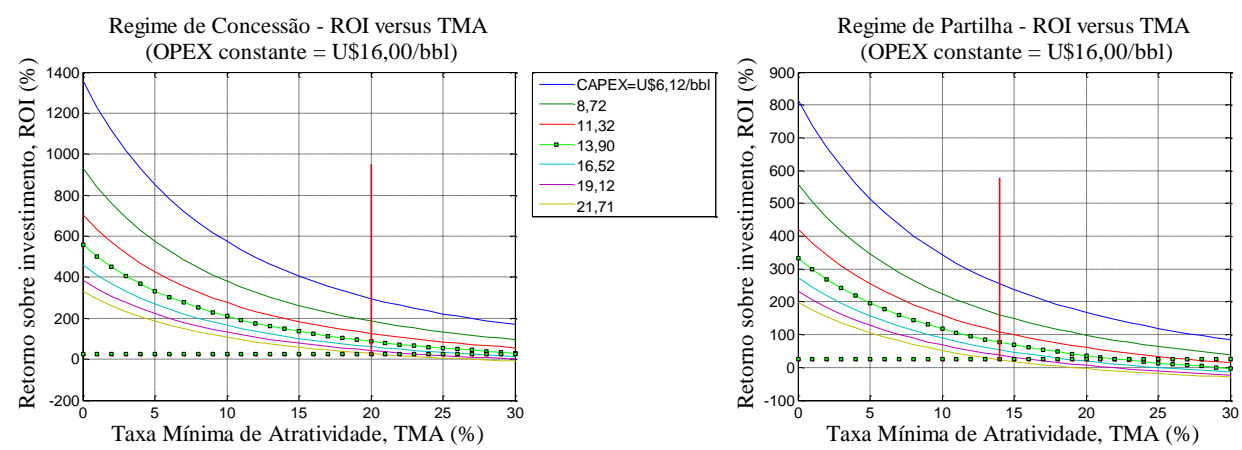

Figura 9: ROI versus TMA (CAPEX variável com OPEX constante).

\section{Curvas de risco (VPL e reserva)}

Considerando as incertezas relativas ao preço da commodity e ao vetor CAPEX e OPEX, podem-se explorar os seguintes resultados: (A) distribuição do VPL; (B) curva de risco e (3) valores AVaR para 4 níveis de confiança. A Figura 10, por exemplo, indica esses resultados para um cenário pessimista. 


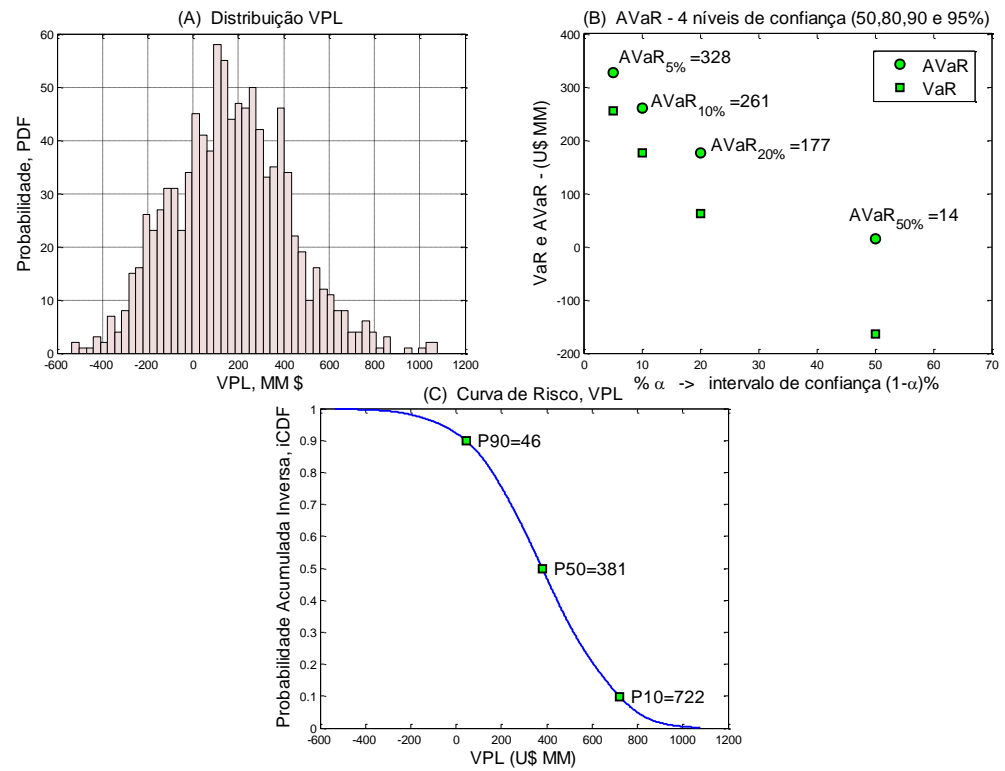

Figura 10: Distribuição do VPL, curva de risco e valor AVaR (cenário pessimista)

Note-se que, apesar do valor médio esperado do VPL ser positivo (166 MMU\$, histograma A), há um risco de 5\% de se perder no mínimo 328MMU\$ nesse projeto (gráfico B). Esta informação, cujo nível de confiança é de 95\%, é impossível de ser obtida com a curva de risco tradicionalmente utilizada em estudos de simulação de reservatório (C). Este é um dos motivos que justifica o uso do conceito $\mathrm{AVaR}$ em complementação à análise de risco com base nos percentis P10, P50 e P90, a exemplo do que se vê nas instituições financeiras de classificação de risco. Ademais, é aquela métrica e não esta que garante coerência com o conceito de aversão ao risco.

Já considerando as incertezas quanto ao desempenho da operadora na execução do projeto (ótimo, normal, ruim), resultam as curvas indicadas na Figura 11.
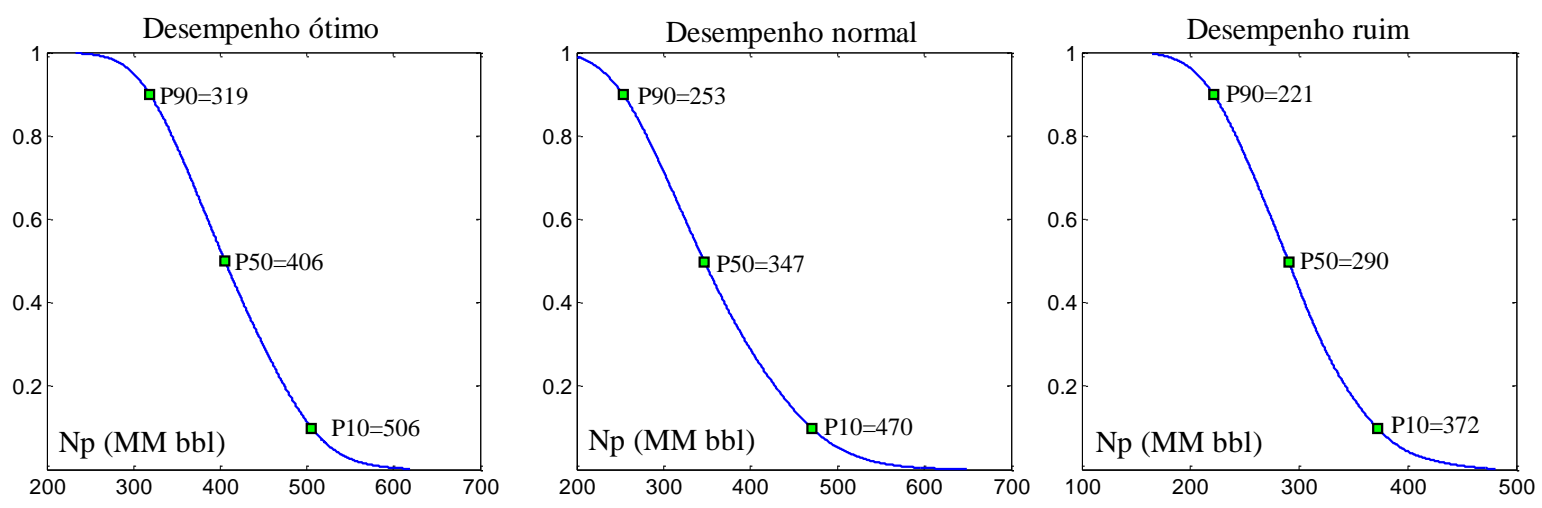

Figura 11: Percentis P10, P50 e P90 para a produção acumulada $N_{p}$ 
Conforme Figura 11, a reserva esperada P50 para uma operadora com desempenho ótimo na condução do projeto é aproximadamente $40 \%$ maior do que o da operadora com desempenho ruim e pelo menos $17 \%$ maior que o da operadora normal. Essa abordagem é útil para mensurar o ganho em reservas de petróleo em caso de antecipação da produção e postergação do declínio de produção em relação ao tempo previsto na estratégia de produção selecionada.

\section{Considerações finais}

Pode-se concluir, resumidamente, que a credibilidade da análise de risco de um projeto de E\&P repousa na capacidade dos processos estocásticos reproduzirem as variáveis fundamentais. A metodologia indicada neste trabalho é simples e a métrica sugerida, AVaR, é consistente com o conceito de aversão ao risco. Os modelos de cópulas e de processos estocásticos propostos aqui têm a capacidade de evidenciar a densidade de eventos extremos, imprescindível para a análise de risco de um projeto de petróleo de alta complexidade com os do pré-sal brasileiro.

Os modelos $\operatorname{GARCH}(2,1)$ e $\operatorname{ARMA}(3,1)$ para a cotação da commodity e TMA, respectivamente, mostraram-se consistentes nos testes retroativos, backtesting. Os processos temporais indicados, como o GARCH, EGARCH e GJR, são mais adequados que os modelos de difusão de Itô (MGB com reversão à média ou com saltos de Poisson), tradicionalmente utilizados em metodologias de análise econômica de projetos de petróleo em condições de incertezas.

Pode-se concluir que, se o objetivo da análise econômica de um projeto de petróleo é capturar os choques havidos no preço da commodity e as dependências assintóticas entre os custos CAPEX e OPEX, os modelos estimados aqui são válidos e, portanto, indicados para outras metodologias de interesse da avaliação econômica de projetos de petróleo. Outros pares de variáveis econômicas podem ser modelados com cópulas bivariadas, além do CAPEX e OPEX. Por exemplo: (i) A taxa mínima de atratividade de projetos e alguma taxa de juros livre de risco, (ii) O preço do óleo e o CAPEX, (iii) A diária de sonda de perfuração de poços e o preço do óleo; (iv) O preço do óleo e a demanda mundial de energia etc.

Por fim, embora sejam abrangentes as possibilidades de aplicação de processos estocásticos para prever o preço da commodity petróleo, esta abordagem deve ser conduzida com parcimônia e com o rigor matemático necessário. Antes de tudo, a teoria econômica deve inspirá-la e os resultados devem ser validados à luz dos teoremas da probabilidade e com os 
testes estatísticos recomendados. Mais ainda, se for possível adotar modelos estocásticos sem que a teoria geral do equilíbrio seja refutada, tanto melhor é o resultado. Sugere-se, ao invés de uso isolado dos modelos da família GARCH, integrá-los com cópulas de variáveis agregadas da economia, conferindo maior consistência para a previsão das variáveis de interesse da análise de risco de projetos de E\&P.

\section{Referências}

ABNT Associação Brasileira de Normas Técnicas. NBR 14.724, de 17.03.2011. Informação e documentação, trabalhos acadêmicos, apresentação. Válida a partir de 17.04.2011. Rio de Janeiro, 2011.

ADELMAN, M. A. The Economics of Petroleum Supply. The MIT Press, Cambridge, MA, 556 p., 1993.

BOLLERSLEV, T. Generalized autoregressive conditional heterocedasticity. Journal of Econometrics 31, 307-27, 1986. crossrefhttp://dx.doi.org/10.1016/0304-4076(86)90063-1

BOX, G.E.P, JENKINS, G. M. Time Series Analysis, Forecasting and Control, Holden Day, San Francisco, 1970.

BRENNAN, M. J., SCHWARTZ, E. S. Evaluating natural resource investments. The Journal of Business 58, pp. 135-157, 1985. crossrefhttp://dx.doi.org/10.1086/296288

BURBIDGE, J., HARRISON, A. Testing for the effects of oil-price rises using vector autoregressions. International Economic Review, 25(1), p. 459-484, 1984. crossref http://dx.doi.org/10.2307/2526209

CLAYTON, D.G. A model for association in bivariate life tables and its applications in epidemiological studies of familial tendency in chronic disease incidence. Biometrika 65, pp. 141-151, 1978. crossrefhttp://dx.doi.org/10.1093/biomet/65.1.141

COPELAND, T., ANTIKAROV, V. Real Options: a practitioner's guide. Texere, New York, 2001.

DIXIT, A. K., Pindyck, R. S. Investment under Uncertainty. Princeton: Princeton University Press, 1994. 468 p., 1994.

ENGLE, R. F.Autoregressive Conditional Heteroskedasticity with Estimates of the Variance of United Kingdom Inflation. Econometrica. Vol. 50, pp. 987-1007, 1982. crossref http://dx.doi.org/10.2307/1912773

FRANK, M.J. On the Simultaneous Associativity of $F(x, y)$ and $x+y-F(x, y)$. Aequationes Mathematicae 19, pp. 194-226, 1979. crossrefhttp://dx.doi.org/10.1007/BF02189866

FRECHET, M. Sur les tableaux de corrélation dont les marges sont données, Annales de l'Université de Lyon, Série A9, 53-77, 1951.

GISSER, M., GOODWIN, T. H. Crude Oil and the Macroeconomy: Tests of Some Popular Notions. Journal of Money, Credit, and Banking, 18, p. 95-103, 1986. crossref http://dx.doi.org/10.2307/1992323 
GUMBEL, E.J. Bivariate Exponential Distributions J. Amer. Stat. Assoc., 55, pp. 698-707, 1960. crossrefhttp://dx.doi.org/10.1080/01621459.1960.10483368

HAMILTON, D. J. Oil and the macroeconomy since World War II, The Journal of Political Economy, 9, 228-248, 1983. crossrefhttp://dx.doi.org/10.1086/261140

HAMILTON, J.D. Time Series Analysis. Princeton University Press Princeton, New Jersey, 799 p., 1994.

KILIAN, L. Not all oil price shocks are alike: disentangling demand and supply shocks in the crude oil market. The American Economic Review, 99(3), p. 1053-1069, 2009. crossref http://dx.doi.org/10.1257/aer.99.3.1053

MCDONALD, R., SIEGEL, D. The Value of Waiting to Invest. Quarterly Journal of Economics 101, pp. 707-728, 1986. crossrefhttp://dx.doi.org/10.2307/1884175

MCNEIL, A. J., FREY R., EMBRECHTS, P. Quantitative Risk Management: concepts, techniques and tools. Princeton University Press, Princeton, NJ, 538 p., 2005.

MEGILL, R. E. An Introduction to Risk Analysis. The Petroleum Publ., Tulsa, 199 pp., 1977

NELSEN, R. B. An Introduction to Copulas New York: Springer, 2006.

NEWENDORP, P. A method for treating Dependencies Between Variables in Simulation Risk Analysis Models. SPE 5581, SPE-AIME, October. Rose, P., 2001. Risk Analysis and Management of Petroleum Exploration Ventures. AAPG, Tulsa, Oklahoma, USA, 1976.

ROCKAFELLAR, R. T., URYASEV, S. Optimization of conditional value-at-risk'. Journal of Risk, Vol 2/Number 3, Spring, pp. 21-41, 2000.

SKLAR, A. Fonctions de repartition $\mu$ a n dimensions et leurs marges, Publications de l'Institut de Statistique de l'Université de Paris, 8, 229-231, 1959.

Recebido em 29.04.14

Aprovado em 19.02.15 